\title{
STABLE THICKENINGS IN THE TOPOLOGICAL CATEGORY
}

\author{
R. L. $\mathrm{CHAZIN}^{1}$
}

ABstract. A thickening, in the topological category, of a complex $K$ is an equivalence class of simple homotopy equivalences $\phi: K \rightarrow M$, where $M$ is a topological manifold with boundary. Here it is shown that for stable thickenings $(\operatorname{dim} M \gg \operatorname{dim} K)$, the set $J(K)$ of stable thickenings is in 1-1 correspondence with homotopy classes of maps of $K$ into BTop.

Wall [1] and Mazur [2] have studied a "functor" of complexes called a thickening. Given a complex $K$, a thickening of $K$ is essentially an $m$-manifold $M$ which is homotopy equivalent to $K$. The set of these, under a suitable equivalence relation, forms a set $J^{m}(K)$. It is clear that this kind of construction can be done in the differentiable, piecewise-linear, or topological categories.

In [1] and [2] it is shown that the stable thickenings of a complex $K$ are a representable functor, i.e. if we denote the stable thickenings of $K$ by $\Im(K)$, then we have $J(K) \approx[K, \mathrm{BO}]$ in the smooth category and $J(K) \approx[K, \mathrm{BPL}]$ in the piecewise-linear category. In this note we establish the analogous result for the topological category. In a subsequent paper, we will give an analogous result for the homotopy category.

1. Definition. We are able to use the same definition as Wall. Let $K$ be a finite complex of dimension $k$ with basepoint $*$, and $\phi: K \rightarrow M$, a simple homotopy equivalence of $K$ into a compact topological manifold-with-boundary of dimension $m, m \geqq k+3$. The notion of simple homotopy equivalence is well defined in the topological category since, by Kirby-Siebenmann [3], every compact topological manifold has the homotopy type of a finite complex.

We require that the basepoint $*$ of $M$ lie in $\partial M$ and that the inclusion $i: \partial M \subset M$ induce an isomorphism $i_{*}: \pi_{1}(\partial M) \rightarrow \pi_{1}(M)$, and that the tangent space of $M$ at $*$ be oriented. Then we say that the pair $(M, \phi)$ defines a pre-m-thickening of $K$.

Define two pre-thickenings $\left(M_{1}, \phi_{1}\right),\left(M_{2}, \phi_{2}\right)$ of $K$ to be equivalent, if there is a (topological) homeomorphism $h: M_{1} \rightarrow M_{2}$, preserving * and the given orientations of the tangent space there, such that

Received by the editors October 22, 1969.

AMS 1970 subject classifications. Primary 57A15; Secondary 55D15, 57C10.

Key words and phrases. Thickening, stable thickening, topological manifold, classification of stable thickenings.

1 Partially supported by NSF Grant GP-7913. 
$h \phi_{1} \sim \phi_{2}:(K, *) \rightarrow\left(M_{2}, *\right)$. An $m$-thickening of $K$ will be such an equivalence class and by $J^{m}(K)$ we denote the set of all such $m$-thickenings.

2. Stable thickenings. As in Wall $[1, \S 5]$ denote by $J(K)$ the stable limit of the inclusions $J^{m}(K) \rightarrow J^{m+1}(K)$. $J(K)$ will be called the set of stable thickenings of $K$.

Given a representative manifold $M$ in $J(K)$ we take its tangent microbundle and pass to the classifying space. Thus we get a classifying map $M \rightarrow \mathrm{BT}$ op and hence a map $K \rightarrow \mathrm{BTop}$, which is basepoint preserving and unique up to homotopy. Thus we have a natural map $\tau(K): \Im(K) \rightarrow[\mathrm{K}, \mathrm{BTop}]$. We can now state the following theorem.

THEOREM 1. For any $K, \tau(K)$ is a bijection.

The remainder of this paper is devoted to the proof of this theorem.

As in the smooth and PL cases, $\tau(*)$ is the homotopy class of the constant map $K \rightarrow$ BTop. Let $\phi: K \rightarrow M_{0}$ be the trivial thickening, i.e. the one corresponding to the map $K \rightarrow$ pt.; $M_{0}$ is parallelizable and hence corresponds to the constant map $K \rightarrow \mathrm{BT}$ op.

The proof that $\tau$ is surjective in the topological category is exactly the same as in the smooth and PL categories; see Wall [1, p. 80]. For completeness, we restate it here.

Let $f: K \rightarrow$ BTop. As Top is the limit of the Top,$f$ can be factored as

$$
K \stackrel{\hat{f}}{\rightarrow} \mathrm{BTop}_{n} \stackrel{j}{\rightarrow} \text { BTop }
$$

where $j$ is inclusion, for some $n$. Thus $f$ induces a bundle over $K$ with fibre $R^{n}$. Since $\phi$ is a homotopy equivalence there is therefore a corresponding bundle $\xi$ over $M_{0}$, a trivial thickening for $K$. Then $\tau(E(\xi))=\pi^{*}(\xi) \oplus \epsilon^{m}$, where $\pi$ is the projection of $\xi$ and $\epsilon^{m}$ a trivial bundle. Let $X$ be the zero section in $\xi$. Then the thickening determined by $\phi$ followed by a map into a compact neighborhood of $X$ is a thickening $\alpha$ such that $\tau(\alpha)=[f]$. The latter assertion follows immediately from the equation

$$
\tau(E(\xi))=\pi^{*}(\xi) \oplus \epsilon^{m}
$$

and the fact that $x$ is a homotopy equivalence.

To prove that $\tau$ is injective we can assume, after stabilizing, that there are thickenings $\left(M_{1}^{m}, \phi_{1}\right)$ and $\left(M_{2}^{m}, \phi_{2}\right)$ with equivalent tangent bundles. We then need the following lemma:

LEMMA 1. Let $\phi: M_{1}^{m} \rightarrow M_{2}^{m}$ be such that $\phi^{*} \tau\left(M_{2}\right) \simeq\left(M_{1}\right)$. Then there is an immersion $\psi: M_{1}^{m} \rightarrow M_{2}^{m}$ such that $\psi \sim \phi$. 
Proof. We use the immersion theorem of Lees [4] or Gauld [5]. For convenience in applying their results we give the proof in the language of microbundles.

Given $\phi$, we construct a representation $\hat{\phi}: \tau\left(M_{1}\right) \rightarrow \tau\left(M_{2}\right)$. This is essentially a map such that the following diagram commutes:

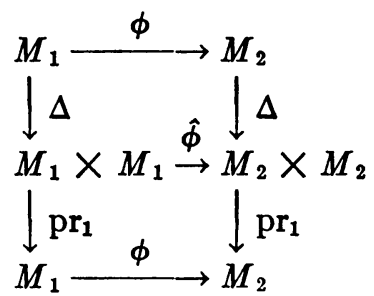

and for which there exist charts

$$
\beta: U \times R^{m} \rightarrow M_{1} \times M, \quad \gamma: U \times R^{m} \rightarrow M_{1} \times M_{2},
$$

such that $\gamma^{-1} \hat{\phi} \beta=1_{U} \times 1_{R^{m}}$.

We now apply Gauld's theorem. This essentially asserts that under certain very mild restrictions, which are satisfied here, homotopy classes of immersions of $M_{1}$ into $M_{2}$ are in 1-1 correspondence with homotopy classes of representations of $\tau\left(M_{1}\right)$ in $\tau\left(M_{2}\right)$. Hence $\phi$ is homotopic to some immersion $\psi$.

Since $m \geqq 2 k+1, \phi_{1}$ is homotopic to an embedding $\phi_{1}^{\prime}$, by Dancis [6]. We can also modify $\psi \phi_{1}^{\prime}$ by a regular homotopy to make it an embedding.

We now wish to compress $M_{1}$ into a neighborhood $U$ of $\phi_{1}^{\prime}(K)$. As there is no satisfactory theory of regular neighborhoods in the topological category, we use Lees' modification [7] of an engulfing theorem due to Newman [8]:

TheOREM 3 (LeEs). Let $Q^{a}$ be an open topological manifold with $q \geqq 5$. Let $U$ be an open subset of $Q$ with $(Q, U)(q-3)$-connected. Suppose that any compact subset of $Q$ lies inside a compact subset $C^{\prime}$ with $\left(Q, Q-C^{\prime}\right)$ 2-connected. Then any compact subset $C$ of $Q$ can be engulfed by $U$, i.e. there is a homeomorphism $h: Q \rightarrow Q$, fixed outside a compact set $C^{\prime \prime}$ with $h(U) \supset C$.

Now attach a collar to $M_{1}$; let $M_{1}^{\prime}=M_{1} \cup \partial M \times I$. Then $M_{1}$ is locally flat in $M_{1}^{\prime}$. A neighborhood $U$ as required by Theorem 3 can be found by removing a suitable small closed subset and using duality or a cellular approximation theorem $[9,7.6 .17]$. Applying Theorem 3, 
we compress $M_{1}$ into $U$, i.e., $1_{M_{1}}$ is isotopic to an embedding of a neighborhood of $M_{1}$ in $M_{1}^{\prime}$, into $U$.

The remainder of the proof now proceeds as in Wall [1]: Since $\psi \phi_{1}^{\prime}$ is an embedding of $K$, we can assume that $\psi$ embeds $\phi_{1}^{\prime} K$ and thus a neighborhood of $\phi_{1}^{\prime} K$. Using the above compression, $\psi$ is isotopic to a map $\psi^{\prime}$ which embeds $M_{1}$ in $M_{2}$. Applying the $s$-cobordism theorem, which holds in the topological category (using Kirby and Siebenmann [3]), we conclude that $M_{1}$ and $M_{2}$ are equivalent thickenings. This completes the proof.

\section{REFERENCES}

1. C. T. C. Wall, Classification problems in differential topology. IV, Topology 5 (1966), 73-94. MR 33 \#734.

2. B. Mazur, Differential topology from the point of view of simple homotopy theory, Inst. Hautes Études Sci. Publ. Math. No. 15 (1963). MR 28 \#4550.

3. R. C. Kirby and L. C. Siebenmann, On the triangulation of manifolds and the hauptvermutung, Bull. Amer. Math. Soc. 75 (1969), 742-749. MR 39 \#3500.

4. J. A. Lees, Immersions and surgeries of topological manifolds, Bull. Amer. Math. Soc. 75 (1969), 529-534. MR 39 \#959.

5. D. Gauld, Mersions of topological manifolds, Thesis, University of California, Los Angeles, Calif., 1969.

6. J. Dancis, Approximations and isotopies in the trivial range, Topology Seminar (Wisconsin, 1965), Ann. of Math. Studies, no. 60, Princeton Univ. Press, Princeton, N. J., 1966. MR $36 \# 7144$.

7. J. A. Lees, Thesis, Rice University, Houston, Tex., 1968.

8. M. H. A. Newman, The engulfing theorem for topological manifolds, Ann. of Math. (2) 84 (1966), 555-571. MR 34 \#3557.

9. E. H. Spanier, Algebraic topology, McGraw-Hill, New York, 1966. MR 35 $\# 1007$.

University of California, Irvine, California 92664 